\title{
Recurrent loss of heterozygosity correlates with clinical outcome in pancreatic neuroendocrine cancer
}

Ben Lawrence ${ }^{1,2}$, Cherie Blenkiron $\mathbb{i D}^{2,3}$, Kate Parker ${ }^{1}$, Peter Tsai ${ }^{3}$, Sandra Fitzgerald ${ }^{3}$, Paula Shields ${ }^{3}$, Tamsin Robb $\mathbb{D}^{3}$, Mee Ling Yeong $^{4}$, Nicole Kramer ${ }^{5}$, Sarah James ${ }^{3}$, Mik Black ${ }^{2,6}$, Vicky Fan ${ }^{7}$, Nooriyah Poonawala ${ }^{7}$, Patrick Yap ${ }^{8}$, Esther Coats ${ }^{1}$, Braden Woodhouse ${ }^{1}{ }^{1}$, Reena Ramsaroop ${ }^{9}$, Masato Yozu ${ }^{10}$, Bridget Robinson ${ }^{11}$, Kimiora Henare ${ }^{12}$, Jonathan Koea ${ }^{13}$, Peter Johnston ${ }^{14}$, Richard Carroll ${ }^{15}$, Saxon Connor ${ }^{11}$, Helen Morrin ${ }^{16}$, Marianne Elston (D) ${ }^{17}$, Christopher Jackson ${ }^{18}$, Papaarangi Reid ${ }^{19}$, John Windsor ${ }^{20}$, Andrew MacCormick ${ }^{20}$, Richard Babor ${ }^{21}$, Adam Bartlett ${ }^{14}$, Dragan Damianovich ${ }^{22}$, Nicholas Knowlton ${ }^{3}$, Sean Grimmond ${ }^{23}$, Michael Findlay ${ }^{1}$ and Cristin Print ${ }^{2,3}$

Pancreatic neuroendocrine tumors (pNETs) are uncommon cancers arising from pancreatic islet cells. Here we report the analysis of gene mutation, copy number, and RNA expression of 57 sporadic well-differentiated pNETs. pNET genomes are dominated by aneuploidy, leading to concordant changes in RNA expression at the level of whole chromosomes and chromosome segments. We observed two distinct patterns of somatic pNET aneuploidy that are associated with tumor pathology and patient prognosis. Approximately $26 \%$ of the patients in this series had pNETs with genomes characterized by recurrent loss of heterozygosity (LoH) of 10 specific chromosomes, accompanied by bi-allelic MEN1 inactivation and generally poor clinical outcome. Another $~ 40 \%$ of patients had pNETs that lacked this recurrent LoH pattern but had chromosome $11 \mathrm{LoH}$, bi-allelic MEN1 inactivation, and universally good clinical outcome. The somatic aneuploidy allowed pathogenic germline variants (e.g., ATM) to be expressed unopposed, with RNA expression patterns showing inactivation of downstream tumor suppressor pathways. No prognostic associations were found with tumor morphology, single gene mutation, or expression of RNAs reflecting the activity of immune, differentiation, proliferative or tumor suppressor pathways. In pNETs, single gene mutations appear to be less important than aneuploidy, with MEN1 the only statistically significant recurrently mutated driver gene. In addition, only one pNET in the series had clearly actionable single nucleotide variants (SNVs) (in PTEN and FLCN) confirmed by corroborating RNA expression changes. The two clinically relevant patterns of $\mathrm{LoH}$ described here define a novel oncogenic mechanism and a plausible route to genomic precision oncology for this tumor type.

npj Genomic Medicine (2018)3:18; doi:10.1038/s41525-018-0058-3

\section{INTRODUCTION}

Pancreatic neuroendocrine tumors (pNETs) are clinically heterogeneous tumors derived from neuroendocrine cells of pancreatic islets, which follow a variable clinical course, but are fatal in $60 \%$ of patients within 5 years. ${ }^{1}$ Effective systemic treatments are emerging including chemotherapies, ${ }^{2}$ radionuclide therapies, ${ }^{3}$ and therapies that target specific molecular changes in tumor cells (e.g., sunitinib, ${ }^{4}$ everolimus ${ }^{5}$ ). Despite recent recognition of profound biological heterogeneity between pNETs, therapeutic decisions are currently made without knowledge of the biological drivers of each individual
pNET, underlining the potential for genomic understanding of these tumors to improve outcomes for patients.

Numerous genomic changes have been observed in welldifferentiated pNETs, including telomeric dysregulation, ${ }^{6}$ copy number $(\mathrm{CN})$ changes, ${ }^{7}$ changes in RNA expression that indicate mTOR pathway activation, germline MEN1 or MUTYH inactivation, ${ }^{9}$ changes in methylation, ${ }^{10}$ and changes to the sequence, methylation, and expression of genes encoding epigenetic modifiers. ${ }^{11}$ Specific mutations in tumor suppressor genes are now accepted as drivers of pNET tumorigenesis (e.g., MEN1, DAXX, ATRX,

\footnotetext{
${ }^{1}$ Discipline of Oncology, Faculty of Medicine and Health Sciences, University of Auckland, Auckland, New Zealand; ${ }^{2}$ Maurice Wilkins Centre hosted by the University of Auckland, Auckland, New Zealand; ${ }^{3}$ Department of Molecular Medicine and Pathology, School of Medical Sciences, Faculty of Medicine and Health Sciences, University of Auckland, Auckland, New Zealand; ${ }^{4}$ Anatomic Pathology Services, Auckland, New Zealand; ${ }^{5}$ LabPlus, Auckland City Hospital, Auckland, New Zealand; ${ }^{6}$ Department of Biochemistry, University of Otago, Dunedin, New Zealand; ${ }^{7}$ Bioinformatics Institute, University of Auckland, Auckland, New Zealand; ${ }^{8}$ Genetic Health Service New Zealand (Northern Hub), Auckland, New Zealand; ${ }^{9}$ Waitemata District Health Board, Auckland, New Zealand; ${ }^{10}$ Histopathology Department, Middlemore Hospital, Auckland, New Zealand; ${ }^{11}$ Canterbury

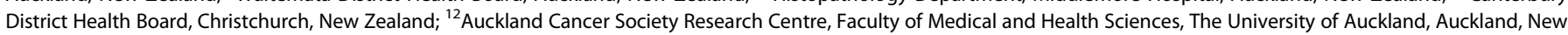
Zealand; ${ }^{13}$ Upper Gastrointestinal Unit, Department of Surgery, North Shore Hospital, Takapuna, Auckland, New Zealand; ${ }^{14}$ Department of Surgery, Auckland District Health

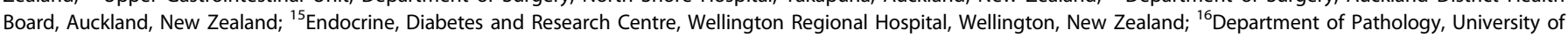
Otago Christchurch, Christchurch, New Zealand; ${ }^{17}$ Waikato Clinical Campus, University of Auckland Department of Medicine, Auckland, New Zealand; ${ }^{18}$ Department of Medicine, Dunedin School of Medicine, University of Otago, Dunedin, New Zealand; ${ }^{19}$ Te Kupenga Hauora Mãori, Faculty of Medical and Health Sciences, University of Auckland, Auckland, New Zealand; ${ }^{20}$ Department of Surgery, University of Auckland, Auckland, New Zealand; ${ }^{21}$ Department of Surgery, Counties Manukau District Health Board, Auckland, New Zealand; ${ }^{22}$ Department of Medical Oncology, Auckland City Hospital, Auckland, New Zealand and ${ }^{23}$ University of Melbourne Centre for Cancer Research, University of Melbourne, Melbourne, Victoria, Australia
}

Correspondence: Cristin Print (c.print@auckland.ac.nz)

These authors contributed equally: Ben Lawrence, Cherie Blenkiron, Kate Parker.

Received: 19 December 2017 Revised: 5 June 2018 Accepted: 22 June 2018

Published online: 20 July 2018 
$V H L, Y Y 1$, and mechanistic target of rapamycin (mTOR) pathway genes ${ }^{9,12,13}$ ). The recognition of such a range of genomic changes in pNETs suggests that each tumor might require multimodal genomic analysis to accurately guide therapeutic choice.

While genomic-enabled oncology has been somewhat successful in many tumor types (e.g., lung, ${ }^{14,15}$ colon, ${ }^{16}$ and ovarian ${ }^{17}$ carcinoma), this is not the case in pNETs. Analyses of tumor tissue to search for predictive biomarkers in pNETs are rare and limited to few markers. For example, although the targets of sunitinib are known (e.g., PDGFR-a, $\beta$, VEGFR-1, 2, 3, FLT3, RET, KIT), the presence of mutations or changes in target expression have been minimally described. ${ }^{18}$ Response to everolimus, an mTOR inhibitor, has been correlated with mTOR pathway protein expression, ${ }^{19}$ but the presence of pathway member gene mutation and RNA expression changes appear in a smaller proportion of patients than expected from clinical trials, and awaits elucidation. Similarly, successful treatment with the alkylating chemotherapy temozolomide occurs in pNETs with low MGMT protein expression in some but not all studies, ${ }^{20-22}$ with MGMT promoter hypermethylation a possible cause. ${ }^{23}$ Despite these correlations suggesting a relationship between response and tumor biology, the relationships are imperfect, and no predictive biomarker is in current use in the clinic. A deeper multimodal description of pNETs is needed to find and understand the biological targets that these agents work on.

Therefore, we undertook pathological examination and deep multimodal genomic analysis of a group of clinically homogenous well-differentiated sporadic pNETs. Our results show that pNETs are dominated by aneuploidy along with MEN1 gene mutation and that the extensive loss of heterozygosity ( $\mathrm{LOH})$ seen in pNETs is linked to dysregulation of RNA expression on the scale of whole chromosomes. Distinct patterns of recurrent chromosome-level aneuploidy relate to clinical outcome and could inform clinical care.

\section{RESULTS}

We analyzed a near-sequential series of 57 sporadic pNETs collected from $53 \mathrm{New}$ Zealand patients along with matched normal tissues. For overall study make-up, see Supplementary Fig. S1; key population characteristics are described in Supplementary Table S1, and individual patient characteristics are described in Supplementary Table S2. Cases selected had a clinical and pathological diagnosis of well-differentiated pNET, expressed at least one of the three neuroendocrine immunohistochemical protein markers (chromogranin A, synaptophysin, or CD56), and were surgically resectable at initial diagnosis. Genomic DNA was analyzed from 47 tumors of 43 patients (including 42 primary tumors) using deep hybridization capture sequencing of 637 genes (578 genes previously associated with cancer, plus an additional 59 genes with published or predicted significance for NETs; Supplementary Table S3). In the 42 primary pNETs analyzed, only a small number of somatic single nucleotide variants (SNVs) and indels with putative functional significance were identified (Fig. 1; Supplementary Table S5). However, further analysis of these sequence data identified substantial $\mathrm{CN}$ gains and losses, in some cases associated with $\mathrm{LoH}$ of large regions of the tumor genome (Fig. 2). RNA expression was then analyzed from 55 tumors of 52 patients (50 primary tumors) using Affymetrix microarrays, informing the interpretation of these somatic mutations and CN DNA changes.

Additional total RNA and mRNA sequence analysis, methylation microarray analysis, and low coverage whole-genome sequencing (WGS) was undertaken for the first 12 tumors in this series for which fresh frozen tissue was available (summarized in Supplementary Table S4). The WGS confirmed the CN changes revealed by the targeted sequencing. In addition, non-negative Matrix Factorization (NMF) mutational signature analysis of the pooled
WGS data from these 12 pNETs revealed a putative $G: C>T: A$ signature (Supplementary Fig. S2a-b). Although sequence depth was insufficient to analyze the 12 tumors individually, a mutational signature similar to that found in the pooled tumors has recently been identified in pNETs. 9

Aneuploidy defines the molecular landscape of pNETs and alters gene expression

Primary pNETs are frequently aneuploid (Fig. 2), with 77\% (30/39) having $\geq 1$ monosomic chromosome (Fig. 3a), 79\% (31/39) having LoH of $\geq 1$ chromosome (leading to loss of one allele of genes on the affected chromosomes, Fig. 3b) and 26\% (10/39) having LoH of $\geq 8$ chromosomes (Fig. $3 \mathrm{~b}$ ). In the aneuploid pNETs, whole chromosome $\mathrm{CN}$ was associated with whole chromosome mean RNA expression, shown for 12 pNETs (001P-012P) that had been analyzed by both expression microarray (Fig. 3c, d) and RNAseq (Fig. 3e, f). Although one pNET (009P) appeared to have a low negative association between whole chromosome $\mathrm{CN}$ and whole chromosome mean RNA expression, this was due to segmental intra-chromosomal CN variation (Fig. 3g), explaining the low correlation observed at whole chromosome level and confirming the observed association between $\mathrm{CN}$ and RNA expression. Similar relationships were seen between whole chromosome $\mathrm{CN}$ and whole chromosome mean RNA expression for all 32 pNETs without intra-chromosomal CN variation (Supplementary Fig. S3a and b). Importantly, this CN-RNA expression relationship was evident for chromosome 11 (Supplementary Fig. S3c), with significantly reduced mean expression of chromosome 11 genes (Mann-Whitney $U$ test $P \leq 0.01$ ) observed in those pNETs with whole chromosome 11 loss (Groups 1 and 2 in Fig. 2) compared to those pNETs with whole chromosome 11 intact.

Patterns of stereotyped aneuploidy and gene expression are associated with clinical behavior

Previous work by Nagano et al. ${ }^{7}$ and Scarpa et al. ${ }^{9}$ identified patterns of chromosomal changes shared between subsets of pNETs. In the pNETs of this study, we found clear CN groups similar to those previously identified and noted strong correlations between $\mathrm{CN}$ group and clinical behavior. Three distinct pNET groups based on CN change emerged (annotated above Fig. 2). In 10 pNETs (labeled Group 1 in Fig. 2), there was a recurrent pattern of LoH affecting the same 10 chromosomes $(1,2,3,6,8,10,11,16$, $21,22)$, which has been previously noted. ${ }^{7,9}$ This idiosyncratic pattern of aneuploidy occurred in the context of somatic MEN1 mutation in nine of 10 tumors, an ATRX or DAXX variant was present in five, with additional PTEN or TP53 mutations present in four. RNA expression analysis showed that MGMT (encoding DNA repair protein 0-6-Methylguanine-DNA Methyltransferase) was generally expressed at lower levels in these 10 tumors than in other tumors ( $t$-test $P<0.01)$. Microarray methylation analysis showed MGMT gene promoter methylation was relatively consistent across pNETs with no significant correlation to expression (data not shown). Therefore, differential MGMT gene methylation, which has been described in other tumor types, ${ }^{24}$ is unlikely to be the dominant mechanism causing lower MGMT RNA expression in this group of pNETs. Instead, one copy of chromosome 10 (the location of MGMT) was lost in all 10 tumors in this cluster, suggesting haploinsufficiency as a more likely mechanism for reduced MGMT expression (Fig. 2).

Tumors in Group 1 had generally less favorable outcomes; four of the 10 tumors in this group had metastasized, this group contained the only three patients who progressed during the study, and all but one tumor had lymphovascular invasion (LVI) on pathological examination. In contrast, tumors in Group 2 (Fig. 2) were characterized by MEN1 mutation and chromosome $11 \mathrm{LoH}$ but no recurrent $\mathrm{LoH}$ of 10 chromosomes. This group had relatively favorable pathological and clinical outcomes; all had low 


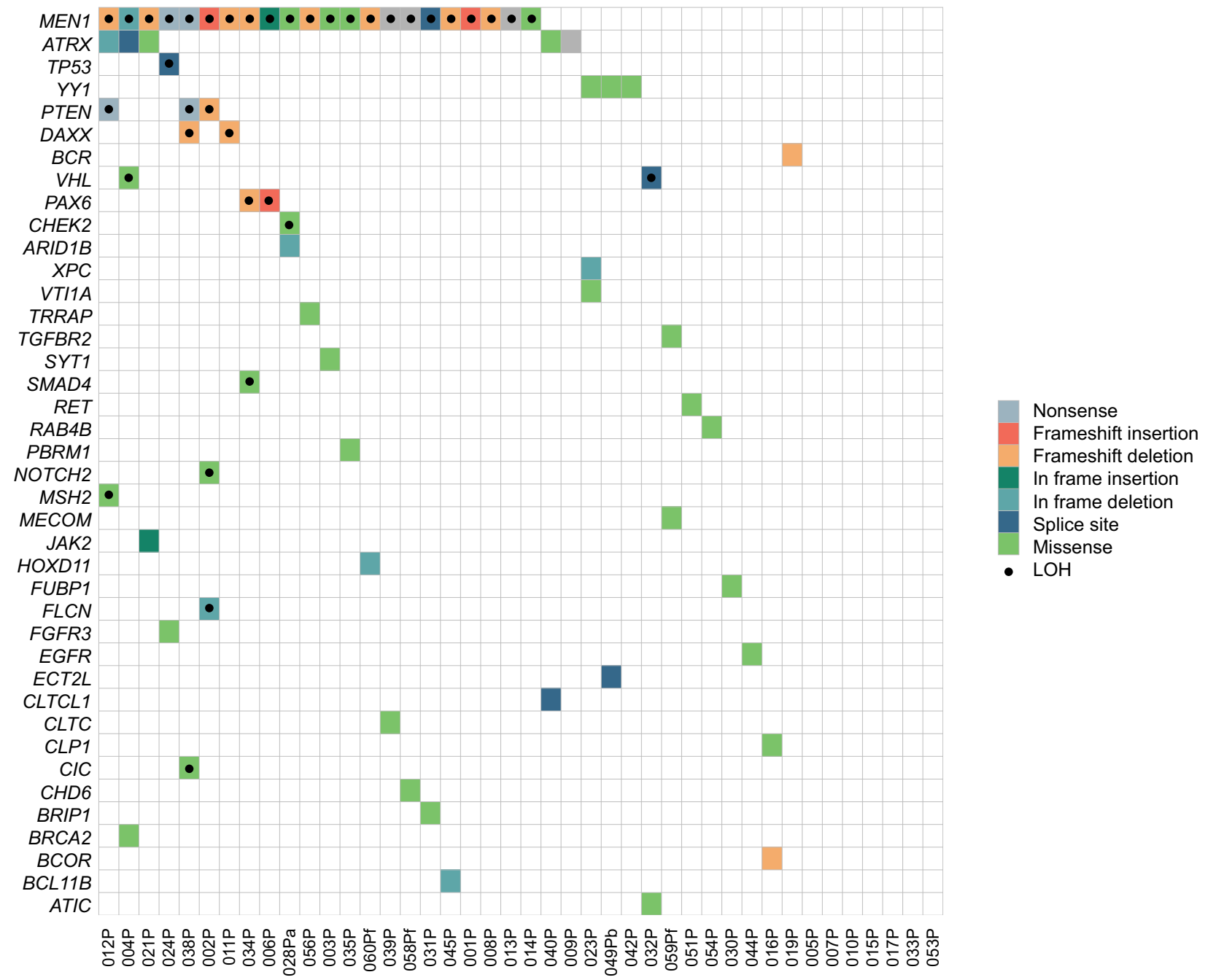

Fig. 1 The mutational landscape of pNETs. Coding region somatic non-synonymous SNVs/indels, large deletions, and intronic mutations within $2 \mathrm{bp}$ of splice sites with any putative functional significance (see Methods) are shown. Tumors are indicated in columns and genes in rows. Colored squares indicate mutation type, with dots indicating that loss of the remaining wild-type allele (LoH) could be confirmed for the locus through changes in both allele frequency of germline heterozygous SNPs and normalized relative regional sequence depth in tumor vs. normal samples. In some tumors, there were no detectable mutations in the 637 genes covered by the targeted sequencing panel

expression of proliferation-associated RNAs, all but one of the 16 tumors in this group were low grade (Ki67 $\leq 2 \%$ ), only three had LVI and none metastasized. Several patients in this group had a clinical history generally associated with inherited cancer predisposition (multiple cancers, multifocal pNETs, and age $\leq 40$ years). Ten of the 14 tumors in this group for which RNA expression data were available had detectable GCG (glucagon) expression. Group 3 (Fig. 2) was characterized by a lack of MEN1 gene mutation, contained tumors with variable patterns of aneuploidy (ranging from none to extensive) and variable clinical outcomes. We are unable to report on progression-free survival due to unavailability of time to progression data. However, proxies for disease severity such as grade and the proportion of patients with metastases were significantly associated with $\mathrm{CN}$ group (Fisher's Exact test $P$ $=0.017$ and 0.018 , respectively).

pNETs have few somatic driver mutations or structural genomic lesions

pNETs have very few detectable somatic variants (Fig. 1, Supplementary Table S5) compared to other tumor types ${ }^{25}$ and only one pNET in this study had more than one variant detected per MB of exons (Fig. 4). Neither large-scale structural variants nor genome duplication were detectable in the data available. However, bi-allelic MEN1 inactivation was common; $81 \%$ of tumors with somatic chromosome $11 \mathrm{LoH}$ had a putative pathogenic variant in the remaining MEN1 allele (Fig. 2). These variants were distributed across the entire MEN1 coding region (Supplementary Fig. S2c). Analysis of MEN1 expression showed that nonsense and frameshift variants were associated with reduced MEN1 RNA abundance (Supplementary Fig. S2d). This suggests that processes such as nonsense-mediated decay may contribute to reduced abundance of MENIN protein in MEN1 mutant tumors, in addition to the pathogenic changes introduced by these mutations altering MENIN protein structure and function.

Methylation analysis showed no clear correlation between MEN1 gene methylation and RNA expression in 15 tumors tested, suggesting that methylation is not an important regulator of MEN1 expression in pNETs.

MutSig analysis ${ }^{25}$ identified MEN1 as the only statistically significant cancer driver gene across this PNET cohort, although previously described variants in a small number of other tumor suppressor genes were seen in multiple tumors (Figs. 1 and 2) including ATRX, DAXX, PTEN, YY1, and VHL. Private variants in 31 other genes were detected in single patients, some of which were 


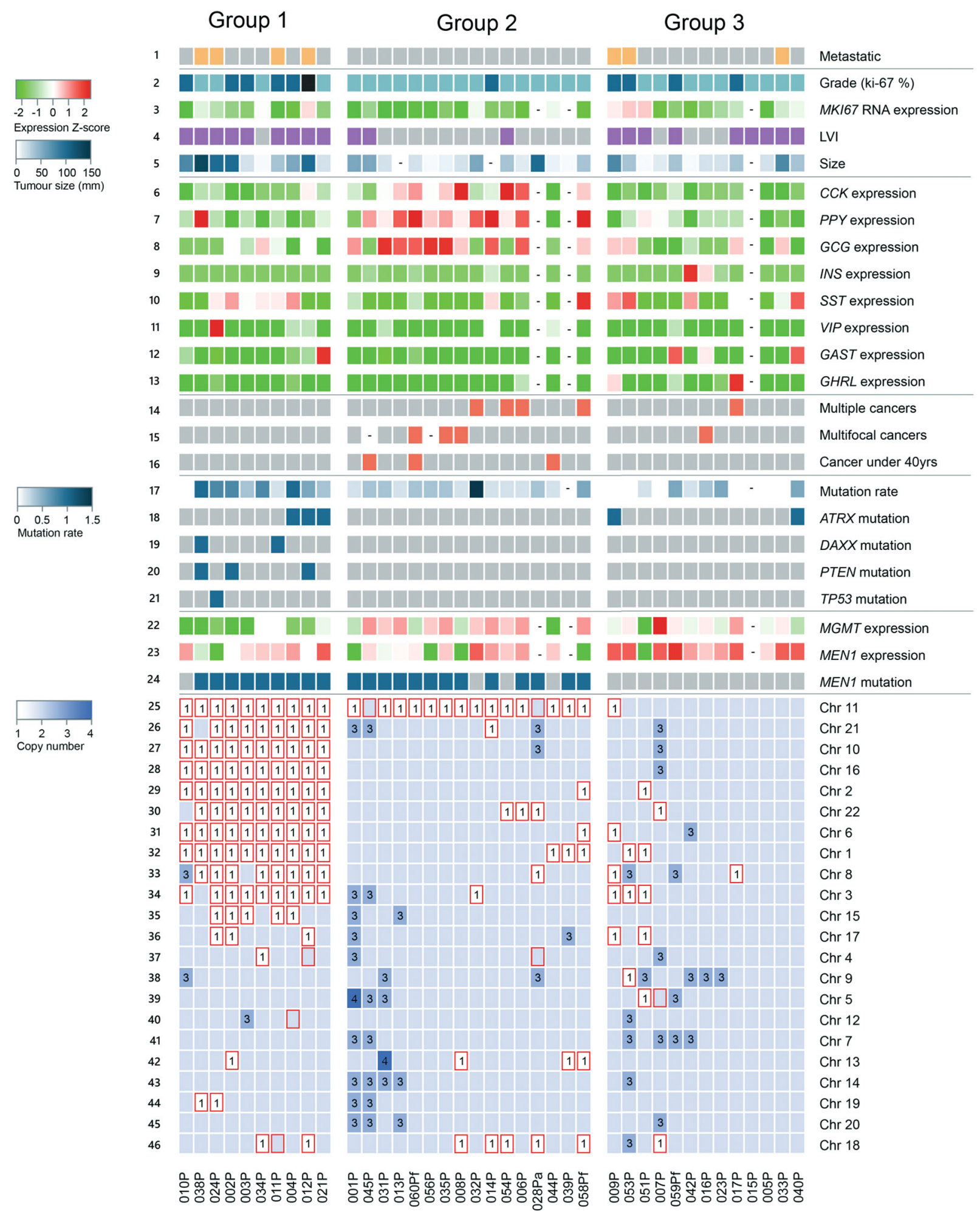

clinically interesting as previously described predictive biomarkers for specific therapies, including bi-allelic inactivation of $M S H 2$ and mono-allelic variants in genes such as RET, JAK2, FGFR3, and BRCA2 (Fig. 1). Although these variants were classified as functionally significant using combinations of variant effect databases (see Methods) and many are considered clinically actionable using current assessment tools (Supplementary Fig. S4), genomic analyses suggested that most were passenger mutations rather than drivers of tumorigenesis. For example, examination of variants in genes encoding tyrosine kinases that have matching small molecule inhibitors found no corroborating JAK2 (patient 021), FGFR3 (patient 024), or RET (patient 051) gene expression/ 
Fig. 2 The genomic landscape of pNETs is dominated by aneuploidy. Tumors are shown in columns and genomic and pathological features in rows. Row 1: metastatic tumors are shown in orange. Row 2: Ki67 $\leq 2 \%$ (defined here as grade 1) is shown in light blue, Ki67 3-20\% (defined as grade 2) in dark blue, and Ki67 >20\% (defined as grade 3) in black. Row 3: MKI67 RNA expression Z-score across tumors (green-red color key to left). Dashes indicate that no expression data were available for specific tumors. Row 4 shows the histological identification of lymphovascular invasion (LVI) in purple, tumors without LVI are colored gray. Row 5 shows tumor size (diameter in mm) on a white-blue scale (white-blue color key to left). Rows 6-13 show expression Z-scores across tumors of the following RNAs (green-red color key to left of row 3): CCK, $P P Y$, GCG, INS, SST, VIP, GAST, and GHRL. Rows 14-16 show multiple cancers of any type in the same individual, multifocal pNETs, and pNETs arising at under 40 years of age, respectively, indicated by red boxes. Row 17 shows the number of functionally significant exonic mutations on a white-blue scale (white-blue color key to left). In rows 18-21, blue squares indicate somatic mutations in the four listed genes. Rows 22 and 23 show the expression of MGMT and MEN1 mRNA (Z-scores, green-red color key to left of row 3). Row 24: Somatic mutations in MEN1 are shown in blue. In rows 25-46, coloring of blocks indicates the dominant inferred CN for each autosome in each tumor based on combined information from: ADTEx analysis, relative somatic read counts at germline heterozygous positions and normalized read counts in $3 \mathrm{~kb}$ tiles across the genome. LoH (irrespective of $\mathrm{CN}$ ) is indicated by red boxes. Unmarked blue boxes indicate an inferred chromosome CN of 2 and numerals indicate $\mathrm{CN}$ when $\mathrm{CN} \neq 2$

pathway changes. However, the mutation pathogenicity of variants in one tumor was corroborated by the RNA expression data-tumor 002P had a somatic frameshift mutation in PTEN (a phosphoinositide 3-kinase (PI3K)/AKT/mTOR signaling inhibitor) as well as a non-frameshift deletion and LoH in the FLCN gene (encodes the mTOR complex 2 inhibitor folliculin). Microarray analysis of the expression of RNAs downstream of PI3K suggested significant activation of PI3K/AKT/mTOR pathways in this tumor, consistent with the mutations (Supplementary Fig. S5).

\section{Comparison of primary and metastatic pNETs}

Two patients in this case series had both primary and metastatic tumors available for analysis (009 and 053) and another had multiple pancreatic primaries that were resected concurrently (patient 028). Supplementary Fig. S6 compares the multiple tumors for these individuals in terms of mutation (Supplementary Fig. S6a) and CN/gene expression/histology (Supplementary Fig. S6b). The two pancreatic tumor regions of patient 028 were indistinguishable in terms of mutation, $\mathrm{CN}$, histology, and gene expression. The pancreatic primary tumor and nodal metastasis of patient 053 were also identical in terms of genome sequence, genome structure, and expression of the SST gene encoding the dominant tumor hormone somatostatin. However, small differences in expression of other hormones between tumor and metastasis were seen.

For individual 009, the primary tumor (009P) and two hepatic metastases (009La and 009Lb; Supplementary Fig. S7) share many genomic features. For example, all three share an ATRX nonsense mutation (Supplementary Fig. S7b) and concordantly the longest telomeres of any pNETs are in this case series (Supplementary Fig. S7c-d). The primary and both metastases also share a low overall mutation rate and moderate aneuploidy (Supplementary Fig. S7ef), moderately high expression of the MKI67 RNA encoding the proliferative protein marker Ki67 (Supplementary Fig. S7g) and high mean expression of the combined Cybersort gene sets ${ }^{26}$ (as a global marker of immune cell infiltration; Supplementary Fig. S7h). In addition, the primary and metastatic tumors of this individual also shared high expression of the RNA encoding ghrelin (Supplementary Fig. S6b); however, neither metastatic tumor carried over the expression pattern of GCG and SST RNAs seen in the primary. Although the interpretation of chromosomal losses and gains was complicated by high and variable stromal content in this patient's tumors, it is clear that the metastases retain the LoH of chromosomes 3 (full), 6 (partial), 8 (partial), and 17 (partial, mixed with amplification) seen in the primary tumor. However, LoH of part of chromosome 11 in the primary tumor was not carried over into the hepatic metastases, yet both metastases gained de novo $\mathrm{LoH}$ of parts of chromosomes 1 and 15 (Supplementary Fig. S8a-c). The regional chromosome $17 \mathrm{LoH} /$ amplification seen in all three tumors was associated with concordant changes in gene expression (Supplementary Fig.
S8d), as was the partial LoH in chromosome 15 acquired by the metastases but absent in the primary (Supplementary Fig. S8e).

Pseudohypoxia determines the expression profiles of some pNETs Tumors from six patients (eight samples) had high expression of a subset of the hypoxia-activated RNAs. These tumors also tended to have more rapid proliferation based on both MKI67 RNA expression (Supplementary Fig. S9a) and immunostaining (seven of these tumors were grade 2 NETs with Ki67 immunostaining in $3-20 \%$ of nuclei). However, further genomic analysis showed that two of the eight tumors had somatic VHL variants with $\mathrm{LoH}$, and tumors from other patients had high $V H L$ gene methylation associated with significantly low VHL RNA expression (Supplementary Fig. S9b). This suggests that in the majority of the pNETs analyzed, tumor hypoxia gene expression profiles are due to pseudohypoxia caused by disrupted VHL function rather than true hypoxia.

Germline variants may become significant in the context of extensive aneuploidy

In our patient series, we were able to exclude with high confidence any functionally relevant germline variants in the following genes previously associated with NETs: MEN1, RET, TSC1, TCS2, PTEN, NF1, CDKN1B, IPMK, MAX, NF1, NTRK1, SDHA, SDHB, $S D H C, S D H D, M U T Y H$, and VHL. However, there were 173 germline variants in 66 genes not traditionally associated with NETs that were predicted to disrupt protein function (Supplementary Table S6). The list of genes affected was significantly enriched for genes associated with DNA repair (GO:0006281, $P=6 \times 10^{-9}$ ) using the PANTHER web tool. Eight of these variants appeared to become unopposed when their remaining normal allele was lost by somatic LoH (Supplementary Table S7). These eight variants had $\sim$ 1:1 ALT:REF allele ratios in germline DNA, and all had somatic LoH in tumor DNA and corresponding tumor ALT:REF allele ratios of $\geq 1.5$. In these tumors, the degree of loss of the remaining normal allele was consistent with the proportion of tumor comprising somatic cells. As an example, tumor 014P had a chr11:108098576_C/G variant in ATM with an ALT:REF allele ratio of 0.9 in the germline but 2.8 in the tumor due to LoH (Fig. 5a). This variant has a population frequency of 0.007 in the ExAc database and leads to a p.Ser49Phe amino acid substitution. Although Clinvar indicated this was a variant of uncertain significance, analysis using IPA and GeneSetDB indicated that numerous RNAs with expression dependent on ATM function were downregulated in this tumor (Fig. 5b), consistent with somatic $\mathrm{LoH}$ exposing a pathogenic germline variant causing somatic loss of ATM activity. 
a

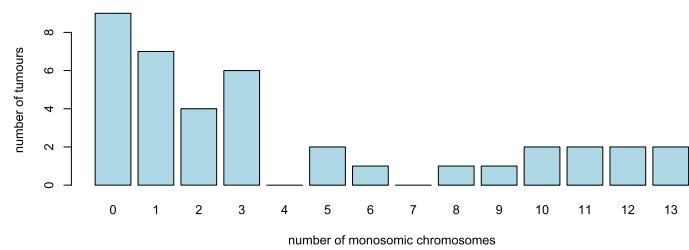

b

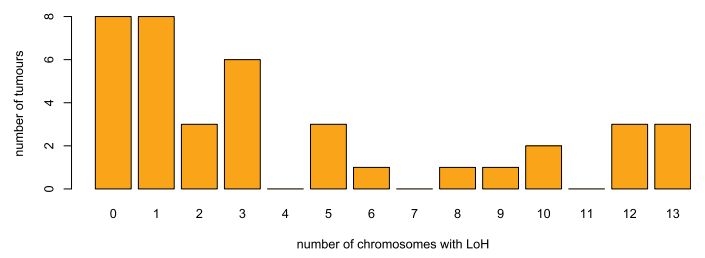

C

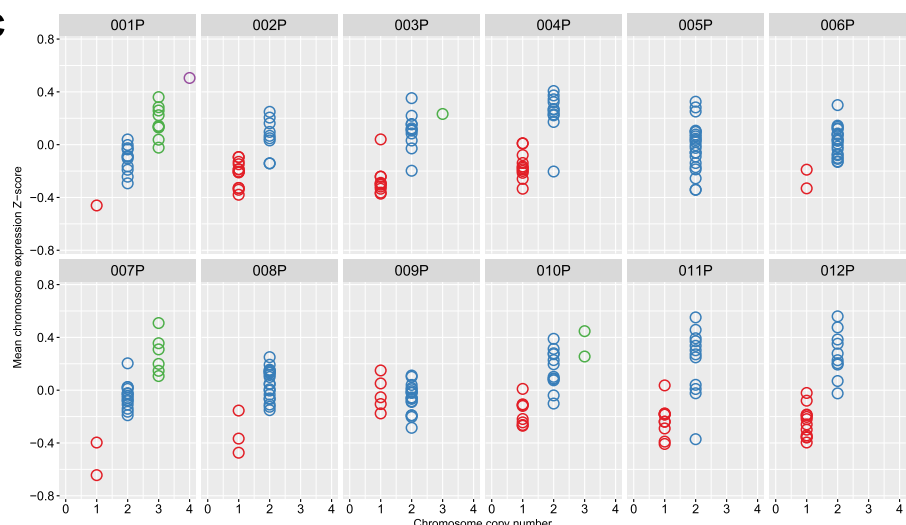

e

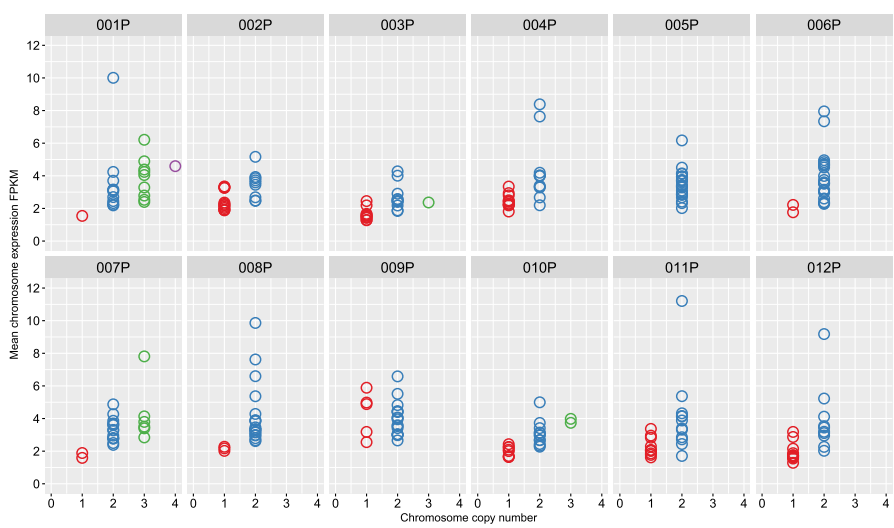

d

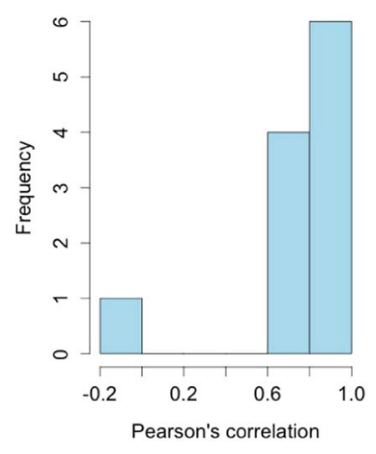

f

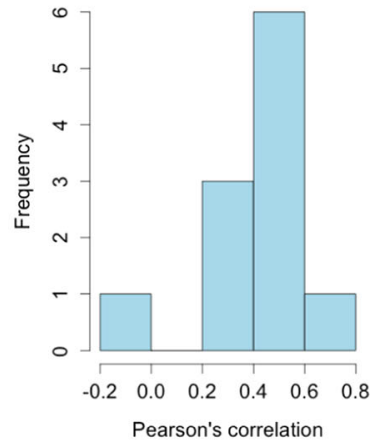

$\mathbf{g}$

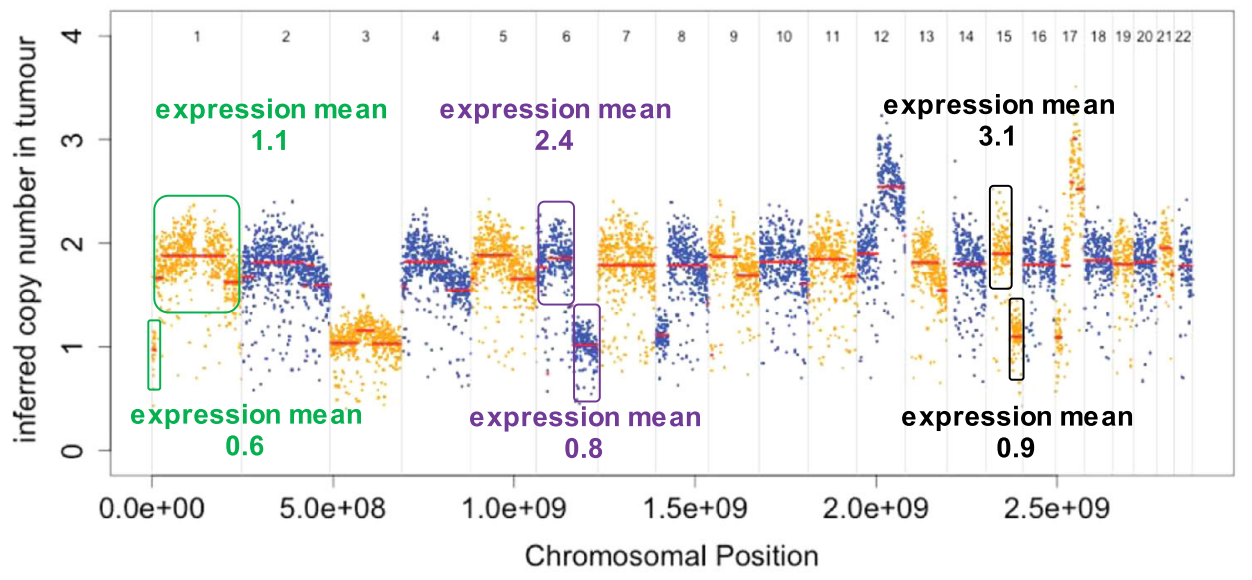

Fig. 3 pNET aneuploidy is extensive but varies between tumors. a Histogram shows the number of monosomic chromosomes (i.e., whole chromosome $\mathrm{LoH}$ with $\mathrm{CN}=1$ ) in individual primary tumors. b Histogram shows the number of chromosomes with LoH (irrespective of $\mathrm{CN}$ ) in individual primary tumors. c-f Graphs compare whole chromosomal CN ( $x$-axis) to mean chromosomal RNA expression based on c microarray data or e RNAseq data ( $y$-axis). Each panel represents a different tumor and each circle represents a different chromosome in that tumor. Histogram of Pearson correlation between $\mathrm{CN}$ and $\mathbf{d}$ microarray RNA expression or $\mathbf{f}$ RNAseq RNA expression in each tumor. $\mathbf{g}$ CN across the genome of the tumor 009P that had negative CN-expression correlation (seen at left of histograms in $\mathbf{b}$ and $\mathbf{d}$ ). This intra-chromosomal analysis confirms the association between CN and RNA expression seen at whole chromosome level in the other pNETs. Chromosomal segments with specific CN aberrations are shown in colored boxes, with mean RNA expression within each of these segments based on fragments per kilobase of transcript per Million mapped reads (FPKM) shown 


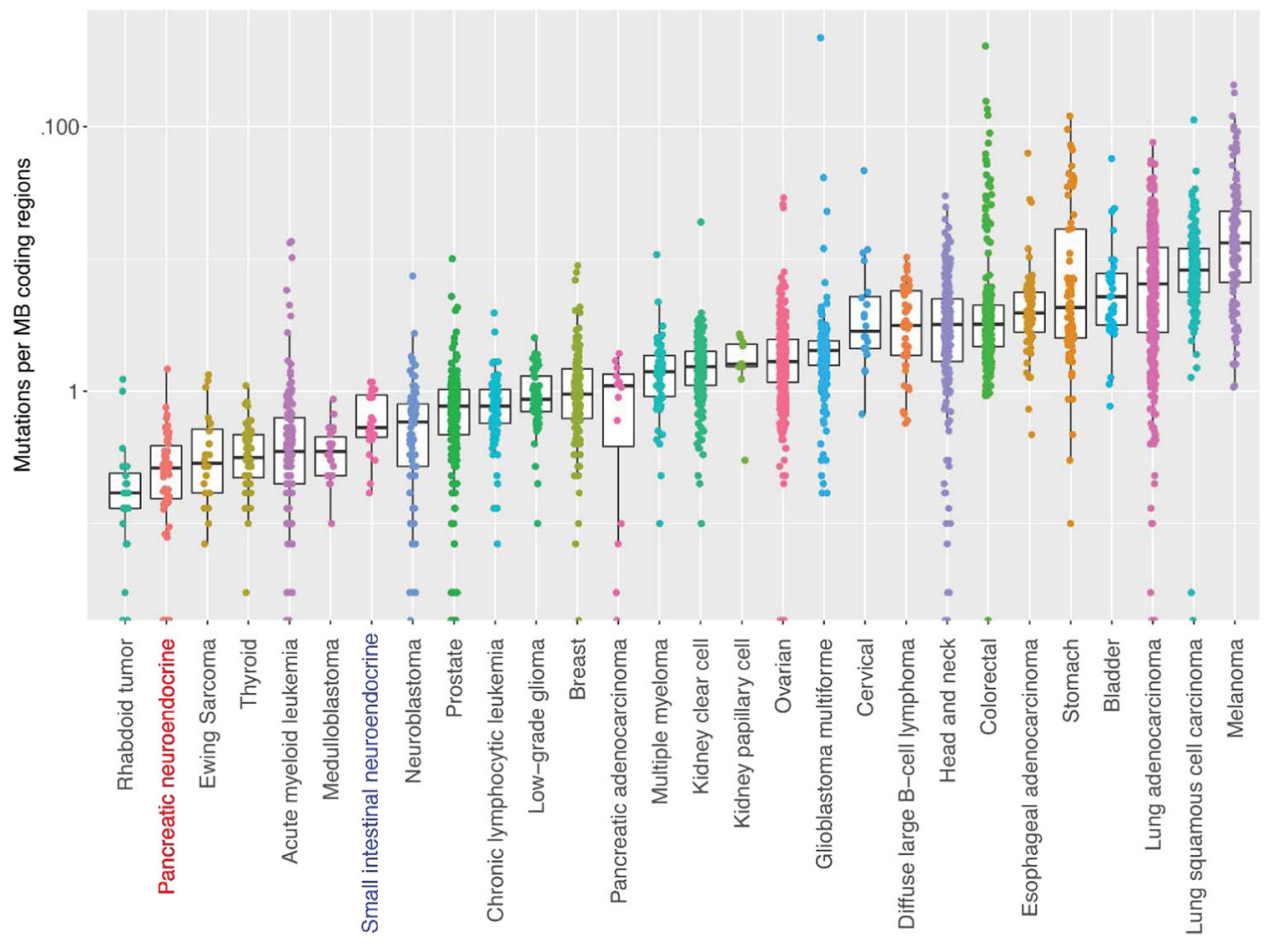

Fig. 4 Number of mutations in pNETs. pNETs have relatively low somatic mutation frequency compared to other tumor types; box plots show the coding region mutation rate of pNETs compared to the coding region mutation rates described by Lawrence et al. ${ }^{25}$ in other tumors analyzed by WES or WGS

Immune, proliferative and hormone expression characteristics of pNETs

The pVAC-Seq neoantigen prediction framework ${ }^{27}$ putatively identified only one tumor (012P) with a mutation capable of generating a potential neoantigen consistent with the patient's HLA haplotype. This low incidence of predicted neoantigens is not surprising, given the low somatic mutation rate in pNETs (Fig. 4). There was no association between grade and somatic variant frequency, suggesting that tumor grade is not determined by single gene events in pNETs.

RNA expression of pancreatic endocrine hormones was examined to identify sub-clinical functioning (e.g., insulinoma, glucagonoma) and "non-functioning" pNETs. Approximately twothirds of pNETs had detectable expression of the RNA encoding at least one hormone, despite the absence of symptoms reported clinically by patients. Expression of the RNAs INS (encodes Insulin) and IAPP (encodes Amylin) appeared correlated, in line with their known co-production by pancreatic islet $\beta$ cells (Supplementary Fig. S10a). The expression of a set of RNAs not usually noted to be co-expressed in the same islet cells (GCG, PPY, and CCK) also appeared correlated. Tumors expressing VIP (encodes Vasoactive Intestinal Peptide) RNA did so exclusively (Supplementary Fig. S10a).

Tumors expressing GHRL (encodes ghrelin, produced by $\varepsilon$ cells) RNA also did so exclusively, and methylation analysis found that high GHRL expression (in the three tumors from patient 009) had low mean methylation of $\mathrm{CpG}$ islands in the GHRL gene promoter, suggesting that dysregulated methylation may have contributed to GHRL expression in this patient (Supplementary Fig. S10b). Differential gene promoter methylation was not associated with RNA expression for any pancreatic endocrine hormones other than ghrelin in the 15 tumors assessed. INS RNA expression was only associated with a clinical diagnosis of 'insulinoma' (biochemically proven hypoglycemia caused by PNET insulin secretion) in a subset of tumors, and in some tumors there appeared to be INS
RNA expression without a documented clinical syndrome (Supplementary Fig. S10a).

\section{DISCUSSION}

Unusual genomic lesions

By combining multiple types of genomic analysis, we have shown that pNETs develop through a range of unusual oncogenic mechanisms. Although more than half of pNETs have biallelic loss of MEN1, the overall frequency of somatic SNVs, indels, and structural DNA variants was low, with small numbers of tumors carrying tumor suppressor variants in ATRX, DAXX, VHL, PTEN, YY1, and PAX6. These variants generally accord with those previously observed in pNETs. ${ }^{9,12,28}$

Rather than mutation, it appears that most pNETs are defined by variable and extensive aneuploidy. For example, approximately $80 \%$ of pNETs in this series had lost a copy of $\geq 1$ chromosome and a recurrent pattern of aneuploidy was observed in some pNETs, which carried LoH of an identical set of 10 chromosomes, therefore affecting thousands of genes. Somatic haploinsufficiency is a plausible mechanism by which this LoH may contribute to pNET development, supported by the striking association we demonstrate between RNA expression and $\mathrm{CN}$ at the level of whole chromosomes and chromosome segments. Given the large number of genes affected on these 10 chromosomes $(\geq 9500)$, it is difficult to identify gene sets or pathways significantly enriched above what could occur by chance. Nevertheless, a range of tumor suppressor genes are now thought to drive tumor development through haploinsufficiency rather than by simple mutation ${ }^{29}$ and aneuploidy can disrupt entire signaling pathways, especially those that depend on precise stoichiometry of protein subunits. ${ }^{30}$ It is possible that development of some pNETs may be driven predominantly by aneuploidy, analogous to chromosome $5 q$ deleted myelodysplastic syndrome in which haploinsufficiency without specific mutation appears to drive the neoplasia. ${ }^{31}$ Understanding the origin, selection in tumor populations and 

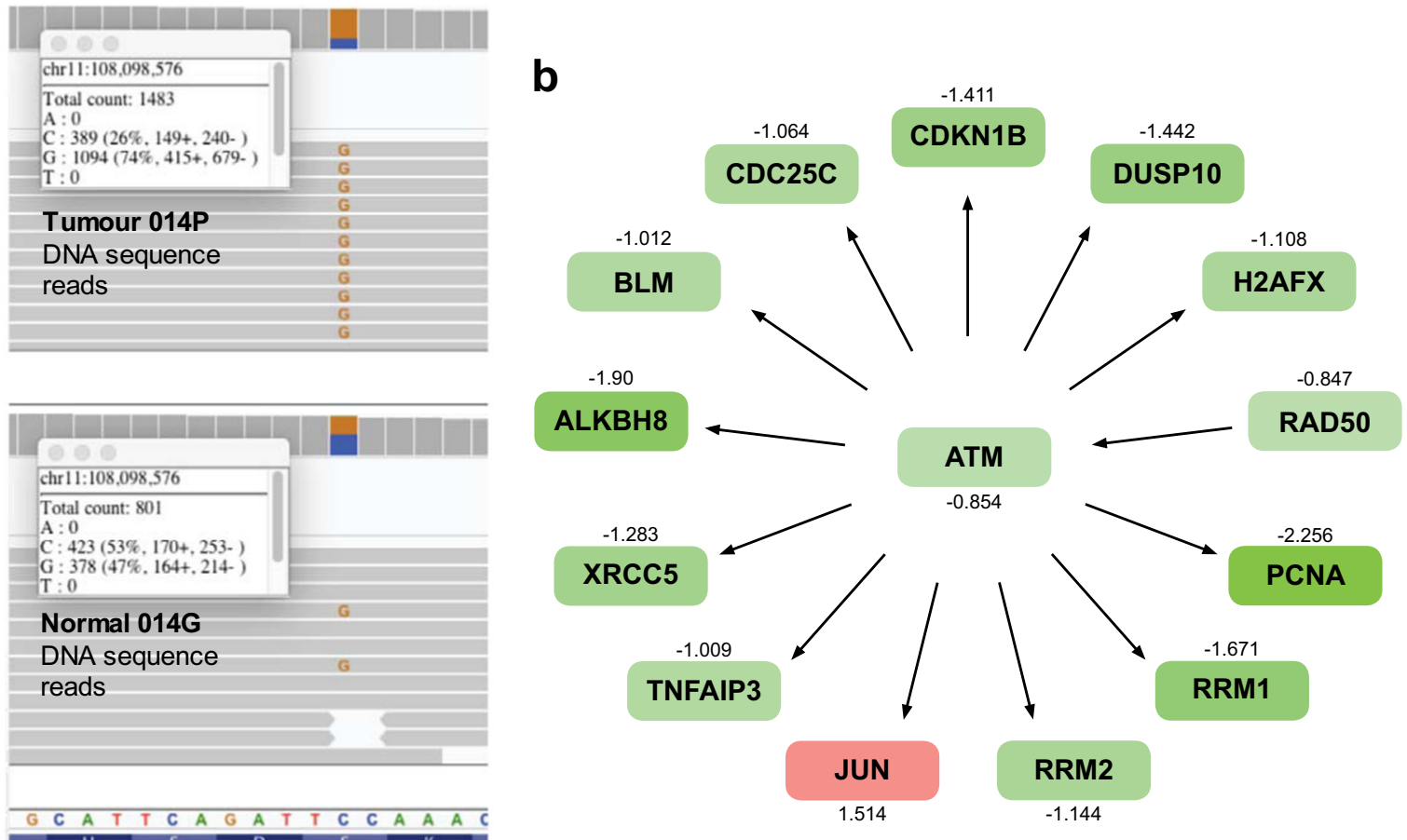

Fig. 5 Somatic LoH may expose germline heterozygous variants. a Example of a germline heterozygous mutation in the ATM locus that becomes unopposed in the tumor 014P due to somatic LoH. b IPA analysis indicates that in this tumor, expression of numerous RNAs that are normally upregulated by the activity of the ATM complex is generally reduced, suggesting reduced ATM function. Shades of red and green indicate the degree of up- and downregulation of RNAs, respectively, with Z-score expression values shown above or below each node

clinical consequences of recurrent aneuploidy, such as we see here, remains a key challenge for modern cancer biology.

Haploinsufficiency is a tenable direct cause for the low MGMT RNA expression in pNETs with the recurrent pattern of 10chromosome loss, since heterozygous $M G M T+/-$ mouse tissues have significantly reduced 0-6-Methylguanine-DNA Methyltransferase activity. ${ }^{32}$ Since low MGMT function is one of the determinants of response to alkylating agents such as temozolomide, pNETs with low MGMT expression may potentially respond to temozolomide therapy, and this aneuploid genotype may have contributed to variations in response to temozolomide in previously published series. ${ }^{33}$ We also show an example where somatic LoH renders a pathogenic heterozygous germline variant in ATM unopposed, thereby inactivating its downstream tumor suppressor pathways (Fig. 5). Careful inspection of the germline in each patient found no evidence of traditional syndromic NETassociated mutations, or the recently recognized MUTYH germline mutations ${ }^{9}$ in our cohort.

Distinct patterns of pancreatic islet hormone expression were seen in the majority of pNETs and may indicate the cell of origin of these tumors. Although RNA expression might not translate into protein expression, high expression of RNAs encoding hormones in most pNETs analyzed suggests that clinicians should be aware of under-diagnosis of subtle secretory syndromes. Interestingly, we also observed one patient with three metastatic tumors with high $G H R L^{34}$ RNA expression and promoter hypomethylation. A recent study of 26 insulinomas found mutations, CN changes and focal allelic imbalances in genes significantly enriched for epigenetic regulators. ${ }^{11}$ However, in the 12 clinically defined insulinomas in our pNET cohort, neither somatic variants nor the genes affected by aneuploidy showed significant enrichment for epigenetic factors listed in EpiFactors database. ${ }^{35}$
Few molecular differences were apparent when we compared two pancreatic primary tumors from one individual, or a primary and nodal metastatic tumor from another. However, while comparing a pancreatic primary and two hepatic metastases of another individual revealed no differences in SNVs or indels, there was clear progression of aneuploidy, with concordant changes in gene expression apparently associated with the metastatic event. Nonetheless, even in this case, the primary tumor and metastases shared molecular features including the same ATRX nonsense mutation accompanied by long telomeres and high expression of an RNA marker of cellular proliferation. In addition, all tumors from this patient shared high expression of immune cell marker genes, suggesting that the drive for tumor immune responses in this patient may be intrinsic to the tumor cells, unaffected by the tissue niche into which metastasis occurred.

Distinct genomic landscapes, putative oncogenic mechanisms, and clinical features of two pNET subsets

By combining $\mathrm{CN}$, somatic variant analysis, and expression analysis, we hypothesize distinct oncogenic mechanisms driving two clinically different subsets of pNETs (Fig. 2), summarized in Fig. 6. The first subset, Group 1, are pNETs with MEN1 mutation coupled with recurrent loss of 10 chromosomes, the cause of which remains unclear. This subset generally had unfavorable grade 2 and 3 histology, all but one patient had LVI and four of the 10 tumors in this group metastasized, and MGMT loss through apparent haploinsufficiency may favor the use of temozolomide. The second subset, Group 2, contained pNETs with MEN1 mutation and chromosome $11 \mathrm{LoH}$ but few other changes in chromosomal CN -none of this group ever went on to metastasize and all but one had favorable low grade histology (Ki67 $\leq 2 \%$ ). In addition, all of this second subset had low expression of proliferation-associated RNAs, 

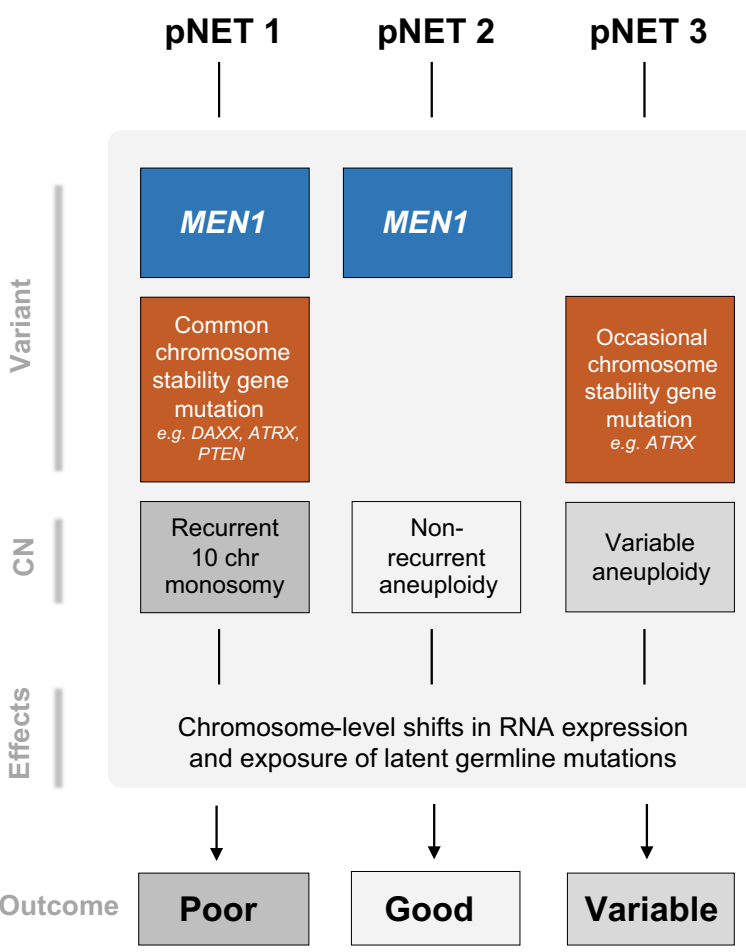

Fig. 6 Integrated genomic, pathological, and clinical categorization of pNETs. Genomic features within pNETs identified three groups: Group 1 generally have MEN1 mutation and chromosome 11 loss, sporadic mutation of genes associated with chromosomal instability, recurrent loss of ten specific chromosomes leading to extensive disruption of gene expression, and reduced MGMT expression. These genomic features are strongly associated with high tumor grade and size, LVI, and more frequent metastases. Group 2 have MEN1 mutation and chromosome 11 loss but no recurrent loss of ten chromosomes. They have universally low tumor grade, size, and LVI, many express GCG RNA, and importantly, this group have no metastases. Group 3 are characterized by no MEN1 mutation, with variable aneuploidy, clinical and pathological features

only three of the 16 tumors in this subset had LVI and most expressed the RNA encoding glucagon. In this subset, the decision to leave tumors un-resected could be considered in the setting of a clinical trial, thus avoiding the complications and long-term morbidity of surgery for these patients.

\section{CONCLUSION}

Aneuploidy appears fundamental for pNET tumorigenesis, possibly by altering gene expression on a global scale and exposing pathogenic germline variants, leading to signaling pathway dysregulation. Simple precision oncology paradigms that match drugs to single gene changes will have low utility in tumors with few mutations such as pNETs. However, the strong genotype-phenotype correlation described here, between two pathognomonic pNET LoH patterns and prognosis, is potentially valuable for clinical decisions affecting approximately two-thirds of pNET patients.

\section{METHODS}

Tumor sample collection and processing

Surgically resected, fresh frozen, and FFPE specimens were collected from the Cancer Society Tissue Bank, University of Otago, NZ, and Auckland Region Hospitals under New Zealand Health and Disability Ethics committee approvals 13/NTA/69 and 13/NTB/173 (for further information on sample handling, see Supplementary Methods). This study used both fresh and archival tumor tissue and was hence conducted under two separate ethical approvals from the Health and Disability Ethics Committees of New Zealand. Written informed consent was obtained from all participants from whom fresh tissue was collected. For archival tissue, to avoid potential ascertainment bias when the donors of more aggressive tumors may have died, due to the relative rarity of this tumor type, and with support from NZ's patient advocacy group for this tumor type, the need for individual informed consent was waived by the Health and Disability Ethics Committees of New Zealand on the condition that these samples were anonymized prior to any analysis being conducted. Matched blood and normal adjacent tissue, where possible $>20 \mathrm{~mm}$ distant, were used as germline controls for the fresh frozen and FFPE cases, respectively. Frozen tissues were processed to isolate genomic DNA (Macheray Nagel; Nucleospin Tissue kit; \#740952) and total RNA (Ambion miRvana RNA isolation kit; Thermo Fisher Scientific; AM1560). FFPE tissues were macro-dissected on slides to maximize tumor cellularity and gDNA and RNA isolated by QIAamp DNA FFPE kit (Qiagen; \#56404) or Ambion RecoverAll kit (Thermo Fisher Scientific; AM1975). Whole blood/buffy coat and dried FTA blood spots $(6 \times 3 \mathrm{~mm}$ punches) were extracted using QIAamp DNA mini kits (\#51102, \#51304). All isolation kits were used as per the manufacturer's instructions. Nucleic acid quality and quantity were determined by Agilent Tapestation and Qubit Fluorometry, respectively.

\section{RNA analysis}

All microarray hybridization and sequencing machine runs for RNAseq were performed as a service by New Zealand Genomics Ltd. For RNAseq, $100 \mathrm{ng}$ RNA from tumors $001 \mathrm{P}-012 \mathrm{P}$ were used as templates to prepare separate total and mRNA libraries using TruSeq Stranded Total RNA Sample Prep kit with Ribo-Zero gold and TruSeq Stranded mRNA Sample Prep kits, respectively. They were sequenced as a multiplex of six samples per HiSeq lane using V3 chemistry, $2 \times 100 \mathrm{PE}$ reads. The total RNA and mRNA sequencing reads were trimmed using cutadapt $^{36}$ v1.9.1 to remove leftover adapters, any reads with Phred score of $<30$, and read pairs where either read was $<50 \mathrm{bp}$ after trimming. Reads were aligned using Bowtie $2^{37}$ with recommended settings. Aligned total RNA reads and mRNA reads were merged using Picard MergeSamFiles before gene and transcripts abundance quantified using RSEM. ${ }^{38}$ Fusion genes were searched for using TopHat-Fusion. ${ }^{39}$

For microarray expression analysis, Affymetrix PrimeView Human Gene Expression arrays were used (perfect-match-only microarrays with $\sim 530,000$ probes covering $\sim 36,000$ transcripts). A total of $100 \mathrm{ng}$ RNA was labeled using the Affymetrix SensationPlus FFPE method according to manufacturer instructions, before hybridization to the gene chips, washing and scanning. QC was performed using Affymetrix Expression Console and in-house $R$ scripts to visualize probe signal distributions relative to control signals. Data for one tumor (015P) were discarded due to very low tumorderived signals relative to spiked-in control signals, and remaining tumor data were quantile normalized in $\mathrm{R}$ using $\mathrm{RMA}^{40}{ }^{40}$ as implemented in the $\mathrm{R}$ 'affy' package. ${ }^{41}$ To remove any systematic FFPE-vs.-fresh frozen sample biases, for each probe, the mean signal within all FFPE tumors was subtracted from each individual FFPE tumor's signal, with an identical adjustment performed within the fresh frozen tumor group. A comparison between the results of RNAseq and microarray analysis revealed relatively high concordance and is shown in Supplementary Fig. S11.

For all visualizations, expression values for each probe set were transformed into $Z$-scores relative to all tumors in the analyzed cohort (by mean centering the data, then expressing the variation above and below the mean on a scale of standard deviation) and all analysis of Probe set differential expression used the LIMMA R package. ${ }^{42}$ Differentially expressed probe sets were tested for enrichment of particular functional categories or pathways using IPA ${ }^{43}$ and GeneSetDB. ${ }^{44}$ Stromal content was estimated from sequence data using ADTEx, ${ }^{45}$ with Immune subtype abundance in the tumors estimated using the Cibersort ${ }^{26}$ and Estimate ${ }^{46}$ methods. To generate Fig. 2, the R Shiny package was used to visualize mRNA expression alongside clinical and pathological information for specific gene sets.

\section{Methylation analysis}

A total of $500 \mathrm{ng}$ of gDNA from each of the 001P-012P, 009La, 009Lb tumors was bisulfite converted as per manufacturer instructions for the EZ DNA methylation kit (Zymo; D5001). Samples were labeled and hybridized as a service by AgResearch Ltd., GenomeNZ section, New Zealand, onto Illumina Infinium Human $450 \mathrm{k}$ methylation arrays. Data were visualized 
and QC performed using the ChAMP package, ${ }^{47}$ which also provided estimates of tumor gene CN. All methylation BeadChips passed QC standards recommended in the ChAMP documentation. Methylation $\beta$ values were subsequently extracted from the idat files with the RnBeads ${ }^{48}$ package in $\mathrm{R}$ using the hg19 human genome assembly and mean aggregation of each of: promoters, CpG islands, and genes. Measurements were filtered using the Greedycut algorithm; background was subtracted using the noob method of the methylumi package before signal intensity normalization using the SWAN method of the minf package. ${ }^{49}$ mRNA expression data (microarray) and gene methylation data were linked through Ensemble gene ID of the respective platform annotation files. A local MySQL database was generated and queried through statistical filters (significance of correlation and level of expression) to identify significantly anti-correlated/correlated methylation and mRNA expression, using the RSQLite package.

\section{DNA sequencing and data analysis}

WGS libraries were generated for tumors 001P-012P using a Rubicon ThruPLEX-FD kit with 3-50 ng of input DNA. For shallow WGS each WGS library was run 1 sample per lane of HiSeq (but split over multiple lanes) with V3 Chemistry $2 \times 100 \mathrm{PE}$ reads. WES enrichment was performed using the Agilent SureSelect V5 + UTR systems on the above libraries and run in a multiplex of 3 per lane as per the WGS analysis. For targeted sequencing, the NimbleGen SeqCap EZ comprehensive cancer panel was used (Roche; \#4000007080-a 4 Mb design that targets 578 cancer-related genes). An additional custom SeqCap EZ choice panel (Roche NimbleGen 06266282001) covering 59 additional genes with a capture space of $354 \mathrm{~Kb}$ was also designed (Custom NET panel) (Supplementary Table S3). This was completed according to manufacturer instructions, and as further described in the Supplementary Methods.

Sequencing reads were quality trimmed using cutadapt v1.9.1 to remove left over Illumina specific adapters and any reads with Phred score of $<30$. Read pairs were removed if either read had an after trimming length of $<50 \mathrm{bp}$. Reads were aligned to UCSC hg19 reference genome using BWA-mem ${ }^{50}$ with default settings and duplicated reads then removed using Picard v2.1.0 MarkDuplicates (http://broadinstitute.github. io/picard/). Aligned reads with minimum mapping quality of 1 were selected using Samtools. ${ }^{51}$ Due to the high sequence depth achieved by target capture, maximum depths were set to 9000 , the Samtools per-Base Alignment Quality calculation was removed, and tumor purity was set to $50 \%$. Finally, the strand filter was removed as it is not applicable to targetcaptured data. The R SomaticSignatures package ${ }^{52}$ was used to identify and plot mutational signatures using non-NMF (Supplementary Fig. S2), with putative origins of mutational signatures based on information in Alexandrov et al..$^{53}$

\section{Variant calling and annotation}

Somatic SNVs and indels were primarily detected using the VarScan $2^{54}$ v2.3.7 somatic workflow. Somatic variants were detected in parallel using Strelka ${ }^{55}$ and $\mathrm{qSNP}^{56}$ without filtering with default settings-all SNVs and indels described in this paper could be detected using all three methods. Neither Varscan's germline nor somatic $p$-value filters were used. VCF files were extensively annotated using PERL scripts modified from ANNOVAR (using ANNOVARs ljb26 database) with additional annotations from The Cancer Genome Interpreter (https://www.cancergenomeinterpreter.org). Variants detected in presence of supplementary reads were additionally annotated with a custom flag in the original VCF file using vcf-annotate in VCFtools. ${ }^{57}$ Somatic variants were then filtered in real time, while visualizing the effects of the filtering, using the $R$ VariantAnnotation and $\mathrm{R}$ Shiny packages. It used the R DNAcopy package ${ }^{58}$ for circular binary segmentation subroutines and for visualization of segmental CN aberrations and corresponding segmental B-allele frequency changes. Postcalling filtering used the following criteria: Normal tissue and tumor read depth at the site of the mutations $\geq 50, \geq 10$ tumor sequences showing the mutation, the site of mutation is not within the Encode Dac Mapability black list, and $\leq 2$ reads corresponding to the mutation were found in the germline sample. All somatic variants that passed these filters were visually validated in IGV. Germline SNVs and indels were detected using the VarScan2 v2.3.7 germline workflow. Mutation plots and lollipop plots in Figs. 1 and 2 and Supplementary Fig. 2c were generated using modifications of the waterfall and lolliplot functions, respectively, of the GenVisR R package. ${ }^{59}$

\section{Coding region mutation rate analysis}

The pNET coding region mutation rate was compared to the rates described by Lawrence et al. ${ }^{25}$ in other tumor types, which had been analyzed by either WGS or WES. Mutation frequencies were calculated in each PNET based on the numbers of coding region mutations found in regions of the genome with $\geq 50 \times$ sequence coverage. Note that PNET mutation frequencies may be overestimated in this analysis since, compared to the WGS or WES analysis used to calculate coding region mutation rates for the other tumor types, the hybridization capture analysis used here is enriched for cancer-associated genes, which may be more likely to carry mutations.

\section{$\mathrm{CN}$ and structural variant analysis}

$\mathrm{CN}$ analysis was performed using the deep targeted sequencing data for all tumors and separately using the WGS data available for tumors 001P-012P. $\mathrm{CN}$ variation was first visualized by counting the number of reads mapped to $3 \mathrm{~kb}$ tiles of the hg19 human genome using bedtools multicov, then analyzed using the DNACopy $\mathrm{R}$ package. For each $3 \mathrm{~kb}$ tile, all raw counts were log2-transformed, normalized using loess splining and log ratios between tumor and normal were calculated, the log ratios were smoothed, segmented (circular binary segmentation), and visualized. B allele frequencies were also analyzed across the tumor genomes to combine with $\mathrm{CN}$ information in order to identify the combination of intra-tumoral heterogeneity and unbalanced chromosomal amplification. To do this required identification of germline heterozygous positions, which was based on: $0.4<$ proportion of ALT reads in germline $<0.6$ and probability $\geq 0.95$ of the observed germline sequence reads being sampled from a population of reads where ALT and REF alleles were equally common, calculated using a binomial distribution. Somatic $\mathrm{CN}$ aberrations were also analyzed in parallel using the Varscan2 $\mathrm{CN}$ pipeline, supplemented by statistical analyses using ADTEx ${ }^{45}$ and Titan, ${ }^{60}$ and for some tumors, CN information from Infinium Methylation BeadChips were analyzed using ChAMP. WGS, WES, and SeqCap aligned BAM files for patients 001-012 were merged using Picard MergeSamFiles before somatic structural variants were analyzed using MANTA, ${ }^{61}$ Delly2, ${ }^{62}$ and GRIDSS $^{63}$ using default settings; in the other tumors, structural variants were analyzed using these three packages from SeqCap data alone.

\section{Data availability}

The data sets generated and analyzed in this study are not freely available out of respect for cultural considerations about genomic data of New Zealand's Mãori people, but the data are available from the European Genome-phenome Archive (https://www.ebi.ac.uk/ega/home; accession number EGAS00001003038) after consideration by a data access committee chaired by the corresponding author.

\section{ACKNOWLEDGEMENTS}

We thank all patients who donated samples and clinical data to our study. We also thank Stefan Bohlander (University of Auckland) for his help with genomic data analysis and interpretation, Ruellyn Cockcroft, Simon Fu, Rebecca Jones and Ole Schmeidel (Auckland DHB), Dean Harris (Canterbury DHB), Richard Issacs (Mid-Central DHB, and Jeremy Rossaak (Bay of Plenty DHB) for clinical input, Siobhan Conroy and Avril Hull of the Unicorn Foundation for coordinating patient input and support, Melissa Firth for input to the Register set up, Maui Hudson and Helen Wihongi for cultural advice and David Jansen for help with translation of key aspects of our project into Te Reo Ma-ori, Malcolm Legget for ongoing advice as Chair of the Translational Medicine Trust, Thierry Lints for thorough review of the manuscript, Ramon Gallego, Aaron Jeffs, Purvi Kakadiya, Rebecca Laurie, John Markham, Les McNoe, John Pearson, Thalanie Purdy, Puja Sharma, Bruce Tsai, Nic Waddell, and Liam Williams for help in the design and interpretation of laboratory studies. This work was financially supported by the Translational Medicine Trust. This work was financially supported by the New Zealand Translational Medicine Trust, Callaghan Innovation, Genesis Oncology Trust, Newmarket Rotary Trusts, Genomics Aotearoa, and the University of Auckland Genomics Into Medicine initiative.

\section{AUTHOR CONTRIBUTIONS}

B.L., C.B., and K.P. contributed equally to this work. B.L. was the co-principal investigator on the project and clinical lead; C.B. coordinated all laboratory work and handling of clinical samples; K.P. led the development of the register of patient information and coordinated the identification, procurement, and annotation of clinical samples; P.T. conducted many of the bioinformatics analyses, supported by V. 
F. and N.P.; M.B. provided bioinformatics input and expert advice; S.F., P.S., T.R., and S. J. conducted DNA and RNA extraction and laboratory analysis; M.L.Y. and N.K. conducted complete pathology review of all samples and, along with R.R.; M.Y. helped with sample identification and procurement; P.Y. provided the clinical genetic review of our analysis; E.C. and B.W. worked on the development of the patient register, B.R. provided clinical annotation of patient samples and clinical input; K.H. and P.R. provided cultural advice and support and helped in the development of a governance framework for the project to support the rights of indigenous persons; J. K., P.J., R.C., S.C., H.M., J.W., A.M., R.B., and A.B. provided help with sample procurement and clinical review of the manuscript; M.E., C.J., and D.D. provided clinical expertise in the interpretation of our analyses; N.K. provided bioinformatics and statistical input,; S.G. provided advice about project design and expert review of the genomic analysis; M.F. was co-principal investigator on the project and provided clinical input; C.P. was the co-principal investigator on the project and provided genomics and bioinformatics expertise.

\section{ADDITIONAL INFORMATION}

Supplementary information accompanies the paper on the npj Genomic Medicine website (https://doi.org/10.1038/s41525-018-0058-3).

Competing interests: The authors declare no competing interests.

Publisher's note: Springer Nature remains neutral with regard to jurisdictional claims in published maps and institutional affiliations.

\section{REFERENCES}

1. Lawrence, B. et al. The epidemiology of gastroenteropancreatic neuroendocrine tumors. Endocrinol. Metab. Clin. North Am. 40, 1-18 (2011).

2. Strosberg, J. R. et al. First-line chemotherapy with capecitabine and temozolomide in patients with metastatic pancreatic endocrine carcinomas. Cancer 117 268-275 (2011).

3. Strosberg, J. et al. Phase 3 trial of (177)Lu-Dotatate for midgut neuroendocrine tumors. N. Engl. J. Med 376, 125-135 (2017).

4. Raymond, E. et al. Sunitinib malate for the treatment of pancreatic neuroendocrine tumors. N. Engl. J. Med 364, 501-513 (2011).

5. Yao, J. C. et al. Everolimus for advanced pancreatic neuroendocrine tumors. $N$. Engl. J. Med 364, 514-523 (2011).

6. Marinoni, I. et al. Loss of DAXX and ATRX are associated with chromosome instability and reduced survival of patients with pancreatic neuroendocrine tumors. Gastroenterology 146, 453-460 e455 (2014).

7. Nagano, Y. et al. Allelic alterations in pancreatic endocrine tumors identified by genome-wide single nucleotide polymorphism analysis. Endocr. Relat. Cancer 14, 483-492 (2007)

8. Missiaglia, E. et al. Pancreatic endocrine tumors: expression profiling evidences a role for AKT-mTOR pathway. J. Clin. Oncol. 28, 245-255 (2009).

9. Scarpa, A. et al. Whole-genome landscape of pancreatic neuroendocrine tumours. Nature 543, 65-71 (2017)

10. Stefanoli, M. et al. Prognostic relevance of aberrant DNA methylation in $\mathrm{g} 1$ and g2 pancreatic neuroendocrine tumors. Neuroendocrinology 100, 26-34 (2014).

11. Wang, $\mathrm{H}$. et al. Insights into beta cell regeneration for diabetes via integration of molecular landscapes in human insulinomas. Nat. Commun. 8, 767 (2017).

12. Jiao, Y. et al. DAXX/ATRX, MEN1, and mTOR pathway genes are frequently altered in pancreatic neuroendocrine tumors. Science 331, 1199-1203 (2011).

13. Cao, Y. et al. Whole exome sequencing of insulinoma reveals recurrent T372R mutations in YY1. Nat. Commun. 4, 2810 (2013).

14. Zhou, C. et al. Erlotinib versus chemotherapy as first-line treatment for patients with advanced EGFR mutation-positive non-small-cell lung cancer (OPTIMAL, CTONG-0802): a multicentre, open-label, randomised, phase 3 study. Lancet Oncol. 12, 735-742 (2011).

15. Soria, J. C. et al. Osimertinib in untreated EGFR-mutated advanced non-small-cell lung cancer. N. Engl. J. Med. 378, 113-125 (2018).

16. Van Cutsem, E. et al. Fluorouracil, leucovorin, and irinotecan plus cetuximab treatment and RAS mutations in colorectal cancer. J. Clin. Oncol. 33, 692-700 (2015).

17. Mirza, M. R. et al. Niraparib maintenance therapy in platinum-sensitive, recurrent ovarian cancer. N. Engl. J. Med. 375, 2154-2164 (2016).

18. Pellat, A. et al. Clinical and biomarker evaluations of sunitinib in patients with grade 3 digestive neuroendocrine neoplasms. Neuroendocrinology 107, 24-31 (2018).

19. Cros, J. et al. Gly388Arg FGFR4 polymorphism is not predictive of everolimus efficacy in well-differentiated digestive neuroendocrine tumors. Neuroendocrinology 103, 495-499 (2016)

20. Cros, J. et al. MGMT expression predicts response to temozolomide in pancreatic neuroendocrine tumors. Endocr. Relat. Cancer 23, 625-633 (2016).
21. Kulke, M. H. et al. O6-methylguanine DNA methyltransferase deficiency and response to temozolomide-based therapy in patients with neuroendocrine tumors. Clin. Cancer Res 15, 338-345 (2009).

22. Cives, M. et al. Analysis of potential response predictors to capecitabine/temozolomide in metastatic pancreatic neuroendocrine tumors. Endocr. Relat. Cancer 23, 759-767 (2016).

23. Campana, D. et al. Correlation between MGMT promoter methylation and response to temozolomide-based therapy in neuroendocrine neoplasms: an observational retrospective multicenter study. Endocrine 60, 490-498 (2017).

24. Gerson, S. L. MGMT: its role in cancer aetiology and cancer therapeutics. Nat. Rev. Cancer 4, 296-307 (2004).

25. Lawrence, M. S. et al. Mutational heterogeneity in cancer and the search for new cancer-associated genes. Nature 499, 214-218 (2013).

26. Newman, A. M. et al. Robust enumeration of cell subsets from tissue expression profiles. Nat. Methods 12, 453-457 (2015).

27. Hundal, J. et al. pVAC-Seq: a genome-guided in silico approach to identifying tumor neoantigens. Genome Med. 8, 11 (2016).

28. Heaphy, C. M. et al. Altered telomeres in tumors with ATRX and DAXX mutations. Science 333, 425 (2011).

29. Davoli, T. et al. Cumulative haploinsufficiency and triplosensitivity drive aneuploidy patterns and shape the cancer genome. Cell 155, 948-962 (2013).

30. Santaguida, S. \& Amon, A. Short- and long-term effects of chromosome missegregation and aneuploidy. Nat. Rev. Mol. Cell Biol. 16, 473-485 (2015).

31. Komrokji, R. S., Padron, E., Ebert, B. L. \& List, A. F. Deletion 5q MDS: molecular and therapeutic implications. Best Pract. Res. Clin. Haematol. 26, 365-375 (2013).

32. Glassner, B. J. et al. DNA repair methyltransferase (Mgmt) knockout mice are sensitive to the lethal effects of chemotherapeutic alkylating agents. Mutagenesis 14, 339-347 (1999).

33. Fine, R. L. et al. Capecitabine and temozolomide (CAPTEM) for metastatic, welldifferentiated neuroendocrine cancers: the Pancreas Center at Columbia University experience. Cancer Chemother. Pharmacol. 71, 663-670 (2013).

34. Corbetta, S. et al. Circulating ghrelin levels in patients with pancreatic and gastrointestinal neuroendocrine tumors: identification of one pancreatic ghrelinoma. J. Clin. Endocrinol. Metab. 88, 3117-3120 (2003).

35. Medvedeva, Y. A. et al. EpiFactors: a comprehensive database of human epigenetic factors and complexes. Database 2015, bav067 (2015).

36. Martin, M. Cutadapt removes adapter sequences from high-throughput sequencing reads. EMBnet. J. 17, 10 (2011).

37. Langmead, B. \& Salzberg, S. L. Fast gapped-read alignment with Bowtie 2. Nat. Methods 9, 357-359 (2012).

38. Li, B. \& Dewey, C. N. RSEM: accurate transcript quantification from RNA-Seq data with or without a reference genome. BMC Bioinformatics 12, 323 (2011).

39. Kim, D. \& Salzberg, S. L. TopHat-Fusion: an algorithm for discovery of novel fusion transcripts. Genome Biol. 12, R72 (2011).

40. Irizarry, R. A. et al. Summaries of Affymetrix GeneChip probe level data. Nucleic Acids Res. 31, e15 (2003).

41. Gautier, L., Cope, L., Bolstad, B. M. \& Irizarry, R. A. affy--analysis of Affymetrix GeneChip data at the probe level. Bioinformatics 20, 307-315 (2004).

42. Smyth, G. K. Linear models and empirical bayes methods for assessing differential expression in microarray experiments. Stat. Appl. Genet. Mol. Biol. 3, Article3 (2004).

43. Kramer, A., Green, J., Pollard, J. Jr \& Tugendreich, S. Causal analysis approaches in ingenuity pathway analysis. Bioinformatics 30, 523-530 (2014).

44. Araki, H., Knapp, C., Tsai, P. \& Print, C. GeneSetDB: a comprehensive meta-database, statistical and visualisation framework for gene set analysis. FEBS Open Bio 2, 76-82 (2012).

45. Amarasinghe, K. C. et al. Inferring copy number and genotype in tumour exome data. BMC Genomics 15, 732 (2014).

46. Yoshihara, K. et al. Inferring tumour purity and stromal and immune cell admixture from expression data. Nat. Commun. 4, 2612 (2013).

47. Morris, T. J. et al. ChAMP: $450 \mathrm{k}$ chip analysis methylation pipeline. Bioinformatics 30, 428-430 (2014).

48. Assenov, Y. et al. Comprehensive analysis of DNA methylation data with RnBeads. Nat. Methods 11, 1138-1140 (2014).

49. Aryee, M. J. et al. Minfi: a flexible and comprehensive Bioconductor package for the analysis of Infinium DNA methylation microarrays. Bioinformatics 30 1363-1369 (2014).

50. Li, H. Aligning sequence reads, clone sequences and assembly contigs with BWAMEM. arXiv 00, 3 (2013). Preprint.

51. Li, H. et al. The Sequence Alignment/Map format and SAMtools. Bioinformatics 25, 2078-2079 (2009).

52. Gehring, J. S., Fischer, B., Lawrence, M. \& Huber, W. SomaticSignatures: inferring mutational signatures from single-nucleotide variants. Bioinformatics $\mathbf{3 1}$, 3673-3675 (2015).

53. Alexandrov, L. B. et al. Signatures of mutational processes in human cancer Nature 500, 415-421 (2013). 
54. Koboldt, D. C. et al. VarScan 2: somatic mutation and copy number alteration discovery in cancer by exome sequencing. Genome Res. 22, 568-576 (2012).

55. Saunders, C. T. et al. Strelka: accurate somatic small-variant calling from sequenced tumor-normal sample pairs. Bioinformatics 28, (1811-1817 (2012)

56. Kassahn, K. S. et al. Somatic point mutation calling in low cellularity tumors. PLoS ONE 8, e74380 (2013).

57. Danecek, P. et al. The variant call format and VCFtools. Bioinformatics 27, 2156-2158 (2011).

58. Venkatraman, E. S. \& Olshen, A. B. DNAcopy: DNA copy number data analysis. $R$ Package Version 1.48 .0 (2016).

59. Skidmore, Z. L. et al. GenVisR: genomic visualizations in R. Bioinformatics 32, 3012-3014 (2016).

60. Ha, G. et al. TITAN: inference of copy number architectures in clonal cell populations from tumor whole-genome sequence data. Genome Res. 24, 1881-1893 (2014).

61. Chen, X. et al. Manta: rapid detection of structural variants and indels for germline and cancer sequencing applications. Bioinformatics 32, 1220-1222 (2016).

62. Rausch, T. et al. DELLY: structural variant discovery by integrated paired-end and split-read analysis. Bioinformatics 28, i333-i339 (2012).
63. Cameron, D. L. et al. GRIDSS: sensitive and specific genomic rearrangement detection using positional de Bruijn graph assembly. Genome Res. 27, 2050-2060 (2017).

(i) Open Access This article is licensed under a Creative Commons adaptation, distribution and reproduction in any medium or format, as long as you give appropriate credit to the original author(s) and the source, provide a link to the Creative Commons license, and indicate if changes were made. The images or other third party material in this article are included in the article's Creative Commons license, unless indicated otherwise in a credit line to the material. If material is not included in the article's Creative Commons license and your intended use is not permitted by statutory regulation or exceeds the permitted use, you will need to obtain permission directly from the copyright holder. To view a copy of this license, visit http://creativecommons. org/licenses/by/4.0/.

(c) The Author(s) 2018 